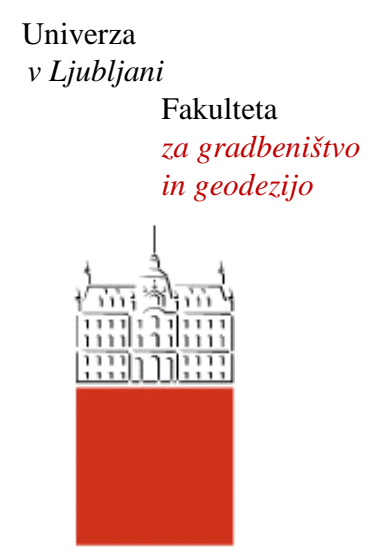

Jamova 2

1000 Ljubljana, Slovenija http://www3.fgg.uni-lj.si//

\section{DRUGG - Digitalni repozitorij UL FGG http://drugg.fgg.uni-lj.si/}

Ta članek je avtorjeva zadnja recenzirana različica, kot je bila sprejeta po opravljeni recenziji.

Prosimo, da se pri navajanju sklicujte na bibliografske podatke, kot je navedeno:
University
of Ljubljana

Faculty of

Civil and Geodetic

Engineering

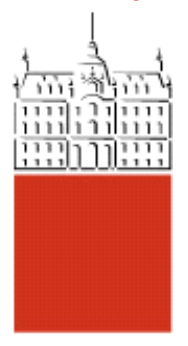

Jamova 2

SI - 1000 Ljubljana, Slovenia

http://www3.fgg.uni-lj.si/

DRUGG - The Digital Repository http://drugg.fgg.uni-lj.si/

This version of the article is author's manuscript as accepted for publishing after the review process.

When citing, please refer to the publisher's bibliographic information as follows:

Brank, B., Carrera, E. 2000. A family of shear-deformable shell finite elements for composite structures. Comput. struct. 76, 1-3: 287-297.

http://www.journals.elsevier.com/computers-and-structures/ 


\title{
A family of shear-deformable shell finite elements for composite structures
}

\author{
Boštjan Brank $^{a, b}{ }^{,}$Erasmo Carrera ${ }^{c}$
}

\author{
aslovenian National Building and Civil Engineering Institute, Dimičeva 12, 1000, Ljubljana, Slovenia

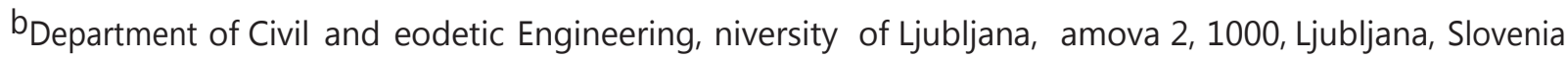 \\ 'Department of Aeronautic and Space Engineering, Politecnico di Torino, Corso Duca degli Abruzzi 24, 10129, \\ Torino, Italy
}

\begin{abstract}
The paper presents some aspects of refined analysis of multilayered composite plates and shells. Refined shell theory is based on a zig-zag (piece-wise linear) variation of displacement field through the thickness, and on a Reissner's mixed variational formulation which allows an introduction of independent transverse shear fields in each layer and an enforcement of the interlaminar shear stress continuity. By relaxing basic assumptions, three additional models of interest are obtained. For the finite element approximation we use a four-noded mixed finite element on the basis of assumed strain variational approach.
\end{abstract}

Keywords: Composite shells; Refined models; Finite elements

\section{Introduction}

In this paper we present a model for composite structures of moderate thickness, which is suitable for the transformation into a relatively simple and efficient multilayered plate and shell finite elements.

The model can be viewed as a refinement of the first-order shear-deformation theory, and requires only $\mathrm{C}^{0}$-continuity of displacements. It avoids dependence of unknown kinematic variables on a number of layers, it satisfies interlaminar equilibrium conditions, and it takes into account discontinuity of derivatives of displacements and transverse shear stresses (with respect to through-thickness coordinate) at layer interfaces [16]. These features are accomplished by two assump- tions: (A) of a zig-zag variation of displacement jield through the thickness of a laminate; and (B) of layer- wise independent quadratic transverse shear stresses. The latter assumption is introduced within the Reiss- ner's mixed variational principle.

The model is in line with works of Murakami [14], Bhaskar and Varadan [3] and Jing and Tzeng [11] who derived linear theories for composite plates, composite cylindrical shells and composite general shells, respect- ively. Carrera [8] and Carrera and Kroeplin[9] reformu- lated the plate theory to be suitable for the finite element approximation and derived some associated linear and geometrically non-linear plate finite el- ements. In this work we generalize the work of Carrera [8] to shells. We present basic points of a general non- linear model, and of three additional formulations obtained by relaxing the preceding basic assumptions. Among these four models, two have been transformed to linear [7] and two to large rotation geometrically nonlinear multilayered shell finite elements $[4,5,10]$. 
Authors are aware that an enormous number of theoretical and computational models have been proposed during the last three decades for an accurate description of linear, non-linear, static or dynamic response of multilayered composite shell-like structures. Many review papers may be found on this topic e.g. [15]. However, we believe that the formulation proposed here has some interesting ingredients, such as: (i) the model has only seven degrees of freedom independently on number of layers; (ii) interlaminar continuity of transverse shear stresses is obtained in a natural way with clear variational background. We also believe that by using recent concepts of modern shell models e.g. [19], the present formulation can be extended to include a reasonable accurate description of throughthe-thickness normal stress.

Refined multilayered shell finite elements developed in this work are four-noded, so-called Assumed Natural Strain (ANS) elements. The ANS concept of Bathe and Dvorkin [1] is used for through-element interpolation of transverse shear strain components, while displacement interpolation is adopted for the membrane and bending parts of the weak form of the boundary value problem.

In what follows, we present basic features of the developed model (and associated submodels) and give some remarks on the finite element approximation. Results of five numerical examples related to linear plates, linear shells and non-linear shells are presented and compared with available solutions. We shortly comment on the effects of transverse shear deformation, zig-zag form of displacement fields and interlaminar shear stress continuity.

\section{Basic concepts of a general model}

\subsection{Multilayered shell kinematic hypothesis}

We consider a laminate of $N_{\text {lay }}$ layers of composite materials as a thin-walled structure. Accordingly, we perform independent parameterization of a reference surface and of a fiber in the thickness direction. Parameterizations are carried out in terms of curvilinear coordinates $\xi:=\left(\xi^{1}, \xi^{2}, \xi \equiv \xi^{3}\right)$ which define material points of the shell. Reference placement of the laminate in space $\mathbb{R}^{3}$ is assumed to be defined as a set

$$
\begin{aligned}
& B:=\left\{\mathbf{X}(\xi) \mid \mathbf{X}(\xi)=\mathbf{M}\left(\xi^{1}, \xi^{2}\right)+\xi \mathbf{T}\left(\xi^{1}, \xi^{2}\right) ;\right. \\
& \quad \xi \in[-h / 2, h / 2]\},
\end{aligned}
$$

where $h$ is the laminate thickness, $\mathbf{M}$ is a point of the reference surface, and $\mathbf{T}$ is initial shell director. The covariant reference metric at a material point $\xi$ is defined in the standard way as $\mathbf{G}=\left[\mathbf{X}_{, i} \cdot \mathbf{X}_{, j}\right]$, where $(\mathrm{o})_{, i}=\partial(\mathrm{o}) /$ $\partial \xi^{i}$.

In this work we assume that the deformed laminate, $S \subset \mathbb{R}^{3}$, is described by a set (assumption $A$ )

$$
\begin{aligned}
S:= & \left\{\mathbf{x}(\xi) \mid \mathbf{x}(\xi)=\mathbf{m}\left(\xi^{1}, \xi^{2}\right)+\xi \mathbf{t}\left(\xi^{1}, \xi^{2}\right)\right. \\
& \left.+\underline{f(\xi) \mathbf{d}\left(\xi^{1}, \xi^{2}\right)}\right\}
\end{aligned}
$$

where $\mathbf{m}$ is a point of the deformed reference surface, $\mathbf{t}$ is shell director in deformed configuration, $f(\xi)$ is a layer dependent zig-zag function which is at layer $K \in$ $\left[1, N_{\text {lay }}\right]$ defined as

$f\left(\xi_{K}\right)=(-1)^{K} \frac{\xi_{K}}{h_{K} / 2}$,

and $\mathbf{d}$ is a vector related to the wrinkling of laminate cross-section. Coordinate $\xi_{K}$ is defined as $\xi_{K} \in\left[-h_{K} /\right.$ $\left.2, h_{K} / 2\right]$, where $h_{K}$ is thickness of layer $K$. With $\mathbf{g}=\left[\mathbf{x}_{, i} \cdot \mathbf{x}_{, j}\right]$ we denote the covariant current metrics at a material point $\xi$

To obtain a stable formulation in the limit of very thin laminate, we introduce inextensibility constraint on the shell-director

$\|\mathbf{T}\|=\|\mathbf{t}\|=\mathbf{1}$.

Its motion may be now expressed by rotation $\mathbf{Q}$ as

$\mathbf{t}=\mathbf{Q} \mathbf{T}$.

$\mathbf{Q}$ is constrained by $\mathbf{s} \cdot \mathbf{T}=0$, where $\mathbf{s}$ is an eigenvector of $\mathbf{Q}$ (also called rotation vector or axial vector). Since $\mathbf{Q}$ describes rotation of a single vector, Eq. (5), it is defined by only two parameters, which may be Eulerlike angles, spherical coordinates, two components of rotational vector, etc. These parameters, along with a type of their update procedure in non-linear analysis, greatly influence on numerical implementation and performance of a finite element. Expression frequently used to define rotation is so-called Rodrigues formula

$\mathbf{Q}=\mathbf{1}+\frac{\sin \|\mathbf{s}\|}{\|\mathbf{s}\|} \mathbf{S}+\frac{1-\cos \|\mathbf{s}\|}{\|\mathbf{s}\|^{2}} \mathbf{S}^{2}$.

In Eq. (6) $\mathbf{S}$ is a skew symmetric matrix associated with an axial vector $\mathbf{s}$ as $\mathbf{S b}=\mathbf{s} \times \mathbf{b}$ for any $\mathbf{b} \in \mathbb{R}^{3}$. Systematic classification of different choices of rotational parameters is presented in recent work of Betsch et al. [2]. Implementation details for Euler-like angles, two components of rotational vector with additive update, and two components of rotational vector with multiplicative update may be found in Brank et al. [4], [5] and [6], respectively.

Vector d, which at material point $\left(\xi^{1}, \xi^{2}\right)$ describes wrinkling of the laminate cross-sections, is treated as an element of the tangent plane to the current con- 
figuration reference surface at point $\left(\xi^{1}, \xi^{2}\right)$. Accordingly, it has to satisfy condition

$\mathbf{d} \cdot\left(\frac{\partial \mathbf{m}}{\partial \xi^{1}} \times \frac{\partial \mathbf{m}}{\partial \xi^{2}}\right)=0$.

By exploiting constraints (4) and (7), we may get an optimal parameterization of the laminate deformation. At a reference surface point $\left(\xi^{1}, \xi^{2}\right)$ we have seven independent kinematic variables, which may be collected in a generalized displacement vector $\mathbf{u}$ as

$\left(\xi^{1}, \xi^{2}\right) \rightarrow \mathbf{u}=\left[\mathbf{v}, \alpha_{1}, \alpha_{2}, d_{1}, d_{2}\right] \in \mathbb{R}^{7}$.

Here $\mathbf{v}\left(\xi^{1}, \xi^{2}\right)=\mathbf{m}\left(\xi^{1}, \xi^{2}\right)-\mathbf{M}\left(\xi^{1}, \xi^{2}\right)$ are displacements of the reference surface point, $\alpha_{1}, \alpha_{2}$ are two rotational parameters, while $d_{1}, d_{2}$ are (refined) variables associated with the wrinkling of laminate cross sections.

\subsection{Strain measures}

The Green-Lagrange strain tensor, defined as $\mathbf{E}=\frac{1}{2}(\mathbf{g}-\mathbf{G})$, may be for the present problem split into two parts: the in-plane part, $\mathbf{E}_{n}$, and the transverse part, $\mathbf{E}_{s}$. The resulting tensors may be written as a sum

$$
\begin{aligned}
\mathbf{E}_{n}= & \mathbf{E}_{n}^{0}+\xi \mathbf{E}_{n}^{1}+f(\xi) \mathbf{E}_{n}^{1, f}+(\xi)^{2} \mathbf{E}_{n}^{2}+\xi f(\xi) \mathbf{E}_{n}^{2, f} \\
& +[f(\xi)]^{2} \mathbf{E}_{n}^{2, f f}, \\
\mathbf{E}_{s}= & \mathbf{E}_{s}^{0}+f^{\prime}(\xi) \mathbf{E}_{s}^{0, f^{\prime}}+f(\xi) \mathbf{E}_{s}^{1, f}+f(\xi) f^{\prime}(\xi) \mathbf{E}_{s}^{1, f, f^{\prime}} \\
& +\xi f^{\prime}(\xi) \mathbf{E}_{s}^{1, f^{\prime}},
\end{aligned}
$$

where $f^{\prime}(\xi)=\mathrm{d} f / \mathrm{d} \xi$ is derivative of Eq. (3), and superscripts 0,1 and 2 relate to the constant, linear and quadratic parts of $\mathbf{E}_{n}$ and $\mathbf{E}_{s}$ tensors, respectively.

Application of the shell-like theory is reasonable only when some restrictions are imposed on the magnitude of the thickness, the curvature and the strains of the laminate. In this manner, we focus on a model based only on the following strain tensors:

$\mathbf{E}_{n}=\mathbf{E}_{n}^{0}+\xi \mathbf{E}_{n}^{1}+f(\xi) \mathbf{E}_{n}^{1, f}$,

$\mathbf{E}_{s}=\mathbf{E}_{s}^{0}+f^{\prime}(\xi) \mathbf{E}_{s}^{0, f^{\prime}}$.

With respect to the first-order shear deformation theory, $\mathbf{E}_{n}^{1, f}$ and $\mathbf{E}_{s}^{0, f^{\prime}}$ represent additional (refined) strains.

Note, that in Eqs. (9) and (10) $\mathbf{E}_{n}^{0}, \mathbf{E}^{1}, f_{n}, \mathbf{E}^{1}, f_{n}, \mathbf{E}_{s}^{0}$ and $\mathbf{E}_{s}^{0, f^{\prime}}$ denote strain tensors, while in what follows, the same notation is related to the corresponding vectors of strain tensor components, i.e.

$$
\begin{aligned}
& \mathbf{E}_{n}^{0}=\left[E_{11}^{0}, E_{22}^{0}, 2 E_{12}^{0}\right]^{T}, \quad \mathbf{E}_{n}^{1}=\left[E_{11}^{1}, E_{22}^{1}, 2 E_{12}^{1}\right]^{T}, \\
& \mathbf{E}_{n}^{1, f}=\left[E_{11}^{1, f}, E_{22}^{1}, 2 E_{12}^{1, f}\right]^{T} \\
& \mathbf{E}_{s}^{0}=\left[2 E_{13}^{0}, 2 E_{23}^{0}\right]^{T}, \quad \mathbf{E}_{s}^{0, f^{\prime}}=\left[2 E_{13}^{0, f}, 2 E_{23}^{0, f}\right] .
\end{aligned}
$$

Explicit form of the above components may be obtained through the definition of Green-Lagrange strain tensor, and Eqs. (10), (1) and (2).

\subsection{Mixed variational principle}

Let us assume, that the laminate is loaded only by an internal and by an external pressure forces on the inner surface, $M^{-}$, and on the outer surface, $M^{+}$, respectively. The Reissner's mixed variational principle for the present problem may then be stated as

$$
\begin{aligned}
\delta I\left(\mathbf{u}, \boldsymbol{\sigma}_{s} ; \delta \mathbf{u}, \delta \boldsymbol{\sigma}_{s}\right)= & \int_{M}\left(\delta \mathbf{E}_{n}^{0} \cdot \mathbf{n}+\delta \mathbf{E}_{n}^{1} \cdot \mathbf{m}+\delta \mathbf{E}_{s}^{0} \cdot \mathbf{q}\right) \mathrm{d} M \\
& +\int_{M}\left(\delta \mathbf{E}_{n}^{1, f} \cdot \tilde{\mathbf{m}}+\delta \mathbf{E}_{s}^{0, f^{\prime}} \cdot \tilde{\mathbf{q}}\right) \mathrm{d} M \\
& +\int_{M}\left\{\sum _ { K = 1 } ^ { N _ { \text { lay } } } \int _ { - h _ { K } / 2 } ^ { h _ { K } / 2 } \delta \boldsymbol { \sigma } _ { s } \cdot \left[-\left(\mathbf{C}_{K}^{s}\right)^{-1} \boldsymbol{\sigma}_{s}\right.\right. \\
& \left.\left.+\mathbf{E}_{s}^{0}+f^{\prime}\left(\xi_{K}\right) \mathbf{E}_{s}^{0, f^{\prime}}\right] \mu \mathrm{d} \xi\right\} \mathrm{d} M \\
& -\left.\int_{M^{-}} \mathbf{p}^{-} \cdot \delta \mathbf{u}\right|_{-h / 2} \mathrm{~d} M^{-} \\
& -\left.\int_{M^{+}} \mathbf{p}^{+} \cdot \delta \mathbf{u}\right|_{h / 2} \mathrm{~d} M^{+}=0
\end{aligned}
$$

where $\mathrm{d} M$ is an element of the reference surface at the reference configuration, $\mu \mathrm{d} \xi=\mathrm{d} B / \mathrm{d} M, \mathbf{p}^{-}$and $\mathbf{p}^{+}$are surface pressure loadings, $\left(\mathbf{C}_{K}^{s}\right)^{-1}$ is part of the layer compliance matrix, and stress resultants and stress couples are given as

$$
\begin{gathered}
\mathbf{n}=\sum_{K=1}^{N_{\text {lay }}} \int_{-h_{K} / 2}^{h_{K} / 2} \boldsymbol{\sigma}_{n} \mu \mathrm{d} \xi, \quad \mathbf{m}=\sum_{K=1}^{N_{\text {lay }}} \int_{-h_{K} / 2}^{h_{K} / 2} \boldsymbol{\sigma}_{n} \xi \mu \mathrm{d} \xi, \\
\mathbf{q}=\sum_{K=1}^{N_{\text {lay }}} \int_{-h_{K} / 2}^{h_{K} / 2} \boldsymbol{\sigma}_{s} \mu \mathrm{d} \xi, \\
\tilde{\mathbf{m}}=\sum_{K=1}^{N_{\text {lay }}} \int_{-h_{K} / 2}^{h_{K} / 2} \boldsymbol{\sigma}_{n} f\left(\xi_{K}\right) \mu \mathrm{d} \xi, \\
\tilde{\mathbf{q}}=\sum_{K=1}^{N_{\text {lay }}} \int_{-h_{K} / 2}^{h_{K} / 2} \boldsymbol{\sigma}_{s} f^{\prime}\left(\xi_{K}\right) \mu \mathrm{d} \xi,
\end{gathered}
$$

where $\boldsymbol{\sigma}_{n}$ and $\boldsymbol{\sigma}_{s}$ are vectors with in-plane components $\left(\sigma^{11}, \sigma^{22}, \sigma^{12}\right)$ and transverse components $\left(\sigma^{13}, \sigma^{23}\right)$ 
of the second Piola-Kirchhoff stress tensor, respectively.

For a layer $K \in\left[1, N_{\text {lay }}\right]$, we may now write vector of transverse stresses, $\boldsymbol{\sigma}_{s}$, as (assumption B)

$\boldsymbol{\sigma}_{s}=\mathbf{P}_{K} \boldsymbol{\beta}_{K}$

where $\mathbf{P}_{K}$ is transverse stress interpolation matrix, and $\boldsymbol{\beta}_{K}$ is vector of layer unknown assumed transverse stresses (stress parameters). $\mathbf{P}_{K}$ and $\boldsymbol{\beta}_{K}$ are defined in accordance with an assumption that the transverse shear stresses vary quadratically through each layer.

The Reissner's mixed variational principle enables us to express layer-wise assumed transverse shear stresses, collected in vector $\boldsymbol{\beta}_{K}$, by generalized displacements. Details of this procedure are given in [7]. Formally we may write

$\boldsymbol{\beta}_{K}=\boldsymbol{\beta}_{K}(\mathbf{u}) \Longrightarrow \boldsymbol{\sigma}_{s}(\mathbf{u})=\mathbf{P}_{K} \boldsymbol{\beta}_{K}(\mathbf{u})$.

During this process, the interlaminar equilibrium (the continuity of interlaminar transverse shear stresses) is obtained by satisfying the following conditions

$\sigma_{(K+1)}^{\alpha 3, \text { bot }}=\sigma_{K}^{\alpha 3, \text { top }} \quad$ if $K \in\left[1, N_{\text {lay }}-1\right]$,

$\sigma_{K}^{\alpha 3, \text { bot }}=\bar{\sigma}^{\alpha 3, \text { bot }} \quad$ if $K=1$,

$\sigma_{K}^{\alpha 3, \text { top }}=\bar{\sigma}^{\alpha, 3 \text { top }} \quad$ if $K=N_{\text {lay }}$,

where $\bar{\sigma}^{\alpha 3 \text {,bot }}$ and $\bar{\sigma}^{\alpha 3 \text {,top }}$ are applied shear stresses at $M^{-}$and $M^{+}$surfaces, respectively, while $\sigma_{K}^{\alpha 3 \text {, bot }}$ and $\sigma_{K}^{\alpha 3}$, top are values of stresses $\sigma^{\alpha 3}$ at bottom and top

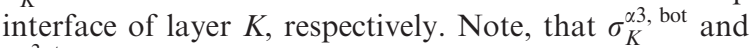
$\sigma_{K}^{\alpha 3, \text { top }}$ are elements of vector $\boldsymbol{\beta}_{K}$. It should be also noted, that by elimination of unknown stress parameters through Eqs. (15) and (16), we obtain the weak form of constitutive equations between the transverse shear stress resultants and the transverse shear strains.

\subsection{Multilayered constitutive relations}

We assume that the material at each layer is orthotropic and elastic. Constitutive relations at layer $K \in$ $\left[1, N_{\text {lay }}\right]$ may be written as $\boldsymbol{\sigma}=\overline{\mathbf{C}}_{K} \mathbf{E}$, where vector of stresses, $\boldsymbol{\sigma}$, vector of strains, $\mathbf{E}$, and block diagonal constitutive matrix, $\overline{\mathbf{C}}_{K}$, are

$\boldsymbol{\sigma}=\left[\boldsymbol{\sigma}_{n}, \boldsymbol{\sigma}_{s}\right]^{T}$,

$\mathbf{E}=\left[\mathbf{E}_{n}^{0}+\xi \mathbf{E}_{n}^{1}+f(\xi) \mathbf{E}_{n}^{1, f}, \mathbf{E}_{s}^{0}+f^{\prime}(\xi) \mathbf{E}_{s}^{0, f^{\prime}}\right]^{T}$,

$\overline{\mathbf{C}}_{K}=\operatorname{Diag}\left[\mathbf{C}_{K}, \mathbf{C}_{K}^{s}\right]$
Details on constitutive matrices $\mathbf{C}_{K}$ and $\mathbf{C}_{K}^{s}$ may be found in standard text books.

Strains, Eq. (11), and stress resultants and stress couples, Eq. (13), may be collected in two vectors as

$\boldsymbol{\epsilon}=\left[\mathbf{E}_{n}^{0}, \mathbf{E}_{n}^{1}, \mathbf{E}_{s}^{0}, \mathbf{E}_{n}^{1, f}, \mathbf{E}_{s}^{0, f^{\prime}}\right]^{T}, \quad \mathbf{N}=[\mathbf{n}, \mathbf{m}, \mathbf{q}, \tilde{\mathbf{m}}, \tilde{\mathbf{q}}]^{T}$

For reasons of simplicity, we have split the shear forces, the stress resultant couples and the strains into two parts. The first part is the same as in the firstorder shear-deformation theory, while the second part arises due to the introduced zig-zag function (3). These quantities are marked as (õ) (stress resultants and stress couples), and as (o $)^{f}$ or (o) ${ }^{f^{\prime}}$ (strains).

Using Eq. (18), multilayered relations between stress resultants and strains may be written as

$\mathbf{N}=\mathbf{H} \boldsymbol{\epsilon}$

where $\mathbf{H}$ is matrix of the following form

$\mathbf{H}=\left[\begin{array}{lllll}\mathbf{C}_{0} & \mathbf{C}_{1} & \mathbf{0} & \mathbf{F}_{10} & \mathbf{0} \\ & \mathbf{C}_{2} & \mathbf{0} & \mathbf{F}_{11} & \mathbf{0} \\ & & \mathbf{Z}_{0} & \mathbf{0} & \mathbf{Z}_{1} \\ & & & \mathbf{F}_{02} & \mathbf{0} \\ \text { symm. } & & & & \mathbf{Z}_{2}\end{array}\right]$

Coefficients of $\mathbf{H}$ are defined as

$\mathbf{C}_{J}=\sum_{K=1}^{N_{\text {lay }}} \int_{-h_{K} / 2}^{h_{K} / 2} \mathbf{C}_{K} \xi^{J} \mu \mathrm{d} \xi$,

$\left(\mathbf{Z}_{0}, \mathbf{Z}_{1}, \mathbf{Z}_{2}\right)=\sum_{K=1}^{N_{\text {lay }}}\left(\mathbf{Q}_{K}^{0}, \mathbf{Q}_{K}^{1}, f^{\prime}\left(\xi_{K}\right) \mathbf{Q}_{K}^{1}\right)$,

$\mathbf{F}_{I J}=\sum_{K=1}^{N_{\text {lay }}} \int_{-h_{K} / 2}^{h_{K} / 2} \mathbf{C}_{K} \xi^{I}[f(\xi)]^{J} \mu \mathrm{d} \xi, \quad J=0,1,2 ;$

$I=0,1$.

Matrices $\mathbf{Q}_{K}^{0}$ and $\mathbf{Q}_{K}^{1}$ are obtained through the functional (12), relation (15) and by satisfaction of interlaminar stress continuity conditions (16). Details of the derivation of these two matrices are given in [7] and references therein.

\section{Special cases}

In this Section we briefly consider special cases (submodels) of the general model presented in Section 2, which we denote (following notation introduced by Carrera [8]) as $R M Z C$ (Reissner-Mindlin Zig-zag Continuous) model. 


\subsection{Submodel 1}

Assumption (B) is not taken into account. Reissner's mixed variational principle is not used, and weak form of equilibrium is satisfied through the principle of virtual work. Transverse shear stresses are obtained with constitutive equations and are therefore constant through each layer. Accordingly, formulation from Section 2 changes in the part where transverse shear constitutive relations are defined. Layer constitutive matrices $\mathbf{Q}_{K}^{0}$ and $\mathbf{Q}_{K}^{1}$ from Eq. (21) are now given as

$\mathbf{Q}_{K}^{J}=\int_{-h_{K} / 2}^{h_{K} / 2} \kappa^{J} \mathbf{C}_{K}^{s} \xi^{J} \mu \mathrm{d} \xi, \quad J=0,1$,

where $\kappa^{J}$ are shear correction factors. With respect to the first-order shear deformation theory the underlined term in Eq. (2) is added to the formulation. We denote this model as $R M Z$ model (Reissner-Mindlin Zig-zag).

\subsection{Submodel 2}

Assumption (A) is relaxed: we drop the underlined term in Eq. (2), so that the variation of displacement field through the thickness is linear. Assumption (B) is completely taken into account. We get a first-order shear-deformation theory within which independent transverse shear stress fields are introduced in each layer in the framework of Reissner's variational principle. The theory does not require any shear correction factors. Since all the terms associated with zig-zag part of Eq. (2) are canceled (i.e. quantities marked with superscripts (õ), (o) $)^{f}$ and $\left.(o)^{f \prime}\right)$, the model has only 5 degress of freedom. Constitutive matrix $\mathbf{H}$ has now the following form,

$\mathbf{H}=\left[\begin{array}{lll}\mathbf{C}_{0} & \mathbf{C}_{1} & \mathbf{0} \\ & \mathbf{C}_{2} & \mathbf{0} \\ \text { symm. } & & \mathbf{Z}_{0}\end{array}\right]$

where the submatrices are defined in Eq. (21). We denote this model as RMC model (Reissner-Mindlin Continuous).

\subsection{Submodel 3}

If assumption (A) is relaxed, so that the variation of displacement field is linear through the thickness, and assumption (B) is not taken into account, we obtain standard first-order shear-deformation theory, which we denote in this work as $R M$ model (Reissner-Mindlin). Quantities marked with superscripts $(\tilde{\mathrm{o}}\},(\mathrm{o})^{f}$ or (o) $f^{\prime \prime}$ are canceled, and transverse shear constitutive matrix has in this case a familiar form

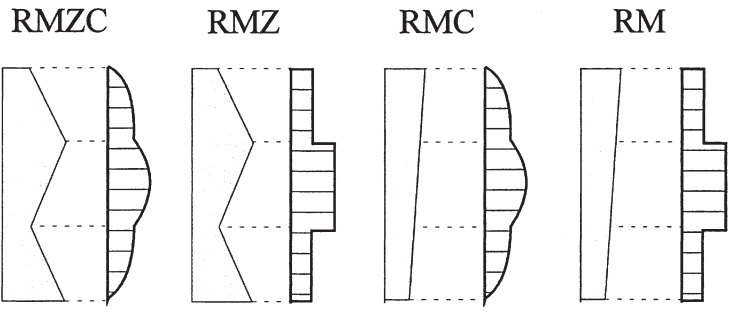

Fig. 1. Through-thickness distribution of displacement field and transverse shear fields for different models. Schematic representation.

$\mathbf{Q}_{K}^{0}=\int_{-h_{K} / 2}^{h_{K} / 2} \kappa \mathbf{C}_{K}^{s} \mu \mathrm{d} \xi$

Assumptions on through thickness distribution of displacements and transverse shear stresses are illustrated on Fig. 1.

\section{Remarks on finite element approximation}

Finite element approximation is based on a fournoded multilayered shell element. Geometric and kinematic fields, their variations and derivatives are interpolated in a standard way by bi-linear interpolation functions $N^{I}$

$\mathbf{M}=\sum_{I=1}^{4} N^{I}\left(\xi^{1}, \xi^{2}\right) \mathbf{M}_{I}, \quad \mathbf{T}=\sum_{I=1}^{4} N^{I}\left(\xi^{1}, \xi^{2}\right) \mathbf{T}_{I}$,

$\mathbf{m}=\sum_{I=1}^{4} N^{I}\left(\xi^{1}, \xi^{2}\right) \mathbf{m}_{I}, \quad \mathbf{t}=\sum_{I=1}^{4} N^{I}\left(\xi^{1}, \xi^{2}\right) \mathbf{t}_{I}$,

$\mathbf{v}=\sum_{I=1}^{4} N^{I}\left(\xi^{1}, \xi^{2}\right) \mathbf{v}_{I}, \quad \mathbf{d}=\sum_{I=1}^{4} N^{I}\left(\xi^{1}, \xi^{2}\right) \mathbf{d}_{I}$,

$\delta \mathbf{t}=\sum_{I=1}^{4} N^{I}\left(\xi^{1}, \xi^{2}\right) \delta \mathbf{t}_{I}, \quad \mathbf{t}_{\alpha}=\sum_{I=1}^{4} N_{, \alpha}^{I}\left(\xi^{1}, \xi^{2}\right) \mathbf{t}_{I}, \ldots$

To avoid shear locking, ANS concept in a form suggested by Bathe and Dvorkin [1] is used for representation of transverse shear components

$$
\begin{aligned}
& \left(2 E_{13}^{0}, 2 E_{13}^{0, f}\right)=\frac{1}{2}\left[1-\xi^{2}\right]\left(2 E_{13}^{0, A}, 2 E_{13}^{0, f, A}\right) \\
& +\frac{1}{2}\left[1+\xi^{2}\right]\left(2 E_{13}^{0, C}, 2 E_{13}^{0, f, C}\right),
\end{aligned}
$$




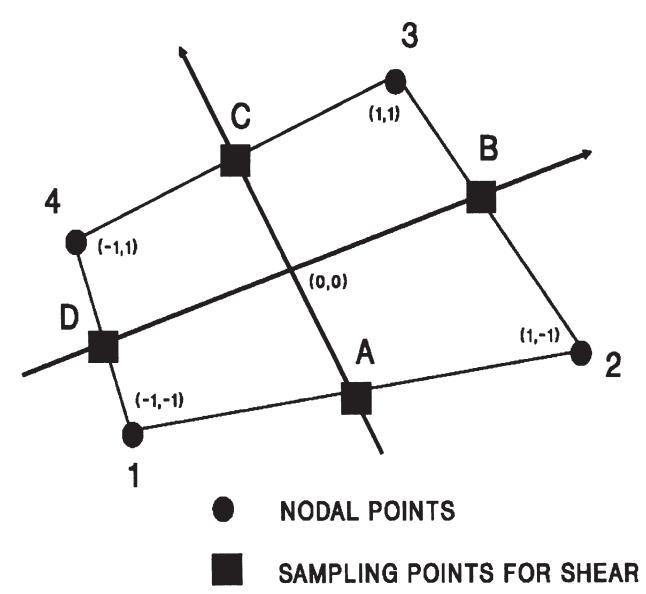

Fig. 2. Four-noded ANS finite element.

$$
\begin{aligned}
& \left(2 E_{23}^{0}, 2 E_{23}^{0, f}\right)=\frac{1}{2}\left[1-\xi^{1}\right]\left(2 E_{13}^{0, D}, 2 E_{13}^{0, f, D}\right) \\
& \quad+\frac{1}{2}\left[1+\xi^{1}\right]\left(2 E_{13}^{0, B}, 2 E_{13}^{0, f, B}\right),
\end{aligned}
$$

where points $A, B, C, D$ are located as shown in Fig. 2 . Since strains at $A, B, C, D$ points are functions of nodal displacements and rotations, explicit evaluation of strains at those points is avoided in the computational process. In accordance with ANS concept (which has its background in $\mathrm{Hu}$-Washizu functional), the variational formulation (12) is modified. Since this is done as in the first-order shear-deformation formulations, the details will not be addressed here. Having this in mind and by using relation (15), functional (12) transforms to the functional with displacements as only unknowns. It may be formally written as

$G(\mathbf{u}, \delta \mathbf{u})=G_{\text {int }}(\mathbf{u}, \delta \mathbf{u})-G_{\text {ext }}(\delta \mathbf{u})=0$,

where $G_{\text {int }}(\mathbf{u}, \delta \mathbf{u})$ is related to internal energy and $G_{\text {ext }}(\delta \mathbf{u})$ is related to external energy.

From the computational point of view, a robust and efficient algorithm with high rate of convergence is required for the solution of non-linear problems. In this work we use the Newton-Raphson method, which requires consistent linearization of functional (27). For linear elastic materials, this results in consistent linearization of the kinematic relations. It should be noted in this respect that the second derivative of the shell director

$\Delta(\delta \mathbf{t})=\left.\frac{\mathrm{d}}{\mathrm{d} \epsilon}\right|_{\epsilon=0}(\delta \mathbf{t})_{\epsilon}$,

where $(\delta \mathbf{t})_{\epsilon}$ is a one parameter curve (which depends on the chosen rotational parameters), is not zero. Further details on linearization will not be discussed here; for linearization aspects of large rotation shell formulations we refer to $[4,5]$. For the present problem interpolation and linearization are not commutative, therefore, consistent linearization is performed after the interpolation. Solution procedure with quadratic rate of asymptotic convergence is attained.

\section{Linear examples}

In this Section we present results of linear analysis of three-layered, cross ply laminated $\left[90^{\circ} / 0^{\circ} / 90^{\circ}\right]$ simply supported square plate and shell panel (see Fig. 3), and make a comparison with the available exact solutions.

\subsection{Example 1: square plate loaded by bi-sinusoidal transverse pressure}

We consider a square plate of length $a$ and thickness $h$, loaded by the bi-sinusoidally distributed pressure $\bar{q}=q \sin (\pi x / a) \sin (\pi y / a)$. Exact solution for this standard test was given in [17]. Material properties are $E_{L} /$ $E_{T}=25.0, \quad G_{L T} / E_{T}=0.5, \quad G_{T T} / E_{T}=0.2, \quad v_{L T}=0.25$,
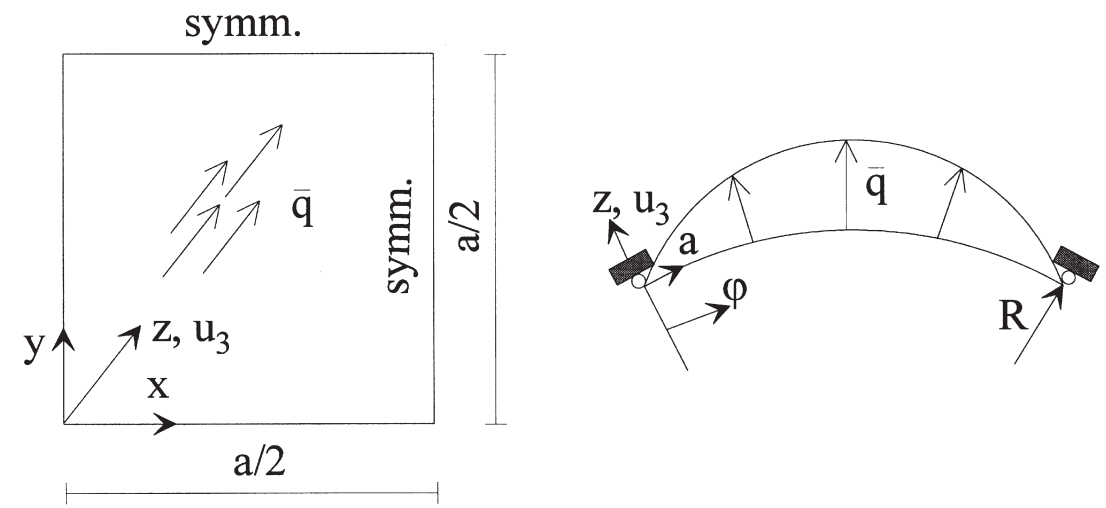

Fig. 3. Geometry of square plate and shell panel. 
Table 1

Square plate: present versus exact solutions; mesh is $16 \times 16$ elements for one quarter of a plate

\begin{tabular}{|c|c|c|c|c|}
\hline$a / h$ & 2 & 4 & 10 & 100 \\
\hline & $\bar{\mu}_{3}$ & & & \\
\hline Exact & 5.1964 & 1.9368 & 0.7370 & 0.4347 \\
\hline RMZC & 5.4081 & 1.9625 & 0.7373 & 0.4336 \\
\hline RMZ & 5.1451 & 1.9034 & 0.7284 & 0.4335 \\
\hline RMs & 5.0576 & 1.7073 & 0.6615 & 0.4327 \\
\hline \multirow[t]{2}{*}{$\mathrm{RMp}$} & 0.4301 & 0.4302 & 0.4302 & 0.4303 \\
\hline & $\bar{\sigma}^{11}$ & & & \\
\hline Exact & $\begin{array}{r}1.3880 \\
-0.0795\end{array}$ & 0.7299 & 0.5590 & 0.5390 \\
\hline RMZC & 0.8742 & 0.6430 & 0.5469 & 0.5365 \\
\hline RMZ & 0.8875 & 0.6394 & 0.5459 & 0.5365 \\
\hline RMs & 0.3380 & 0.4041 & 0.0497 & 0.5360 \\
\hline \multirow[t]{2}{*}{$\mathrm{RMp}$} & - & - & - & 0.5364 \\
\hline & $\bar{\sigma}^{12}$ & & & \\
\hline Exact & $\begin{array}{r}-0.0863 \\
0.0673\end{array}$ & 0.0467 & 0.0275 & 0.0214 \\
\hline RMZC & 0.0711 & 0.0449 & 0.0273 & 0.0213 \\
\hline RMZ & 0.0690 & 0.0442 & 0.0271 & 0.0213 \\
\hline RMs & 0.0354 & 0.0307 & 0.0240 & 0.0212 \\
\hline $\mathrm{RMp}$ & - & - & - & 0.0212 \\
\hline
\end{tabular}

where following usual notations, $L$ defines the fiber direction, $T$ the transverse direction, and $v_{L T}$ is the major Poisson's ratio. The thickness of $0^{\circ}$ layers (with $L$ having direction of $x$ ) is $h / 4$ and the thickness of $90^{\circ}$ layer is $h / 2$. For comparison, the non-dimensional displacement and stresses are introduced as $\bar{u}_{3}=u_{3} 100 E_{T} h^{3} /\left(q a^{4}\right),\left(\bar{\sigma}^{11}, \bar{\sigma}^{12}\right)=\left(\sigma^{11}, \sigma^{12}\right) h^{2} /\left(q a^{2}\right)$. In Table 1 we present $\bar{u}_{3}$ at $(a / 2, a / 2), \bar{\sigma}^{11}$ at the closest integration point to $(a / 2, a / 2, h / 2)$, and $\bar{\sigma}^{12}$ at the closest integration point to $(0,0,-h / 2)$. The same notation is used as in Section 3. RMs is first-order shear-deformation formulation with shear correction factor $\kappa=5 /$ 6 , while RMp denotes results obtained with $\kappa=10^{5}$. In Fig. 4, the distribution of the transverse shear stresses $\sigma^{13}$ (at the closest integration point to $(0, a / 2)$ ) is presented for ratio $a / h=4$. Q4 denotes present results, 3D exact solution, and Q9 solution of nine-noded RMZC plate element from [8]. RMs results for $\sigma^{13}$ (obtained from constitutive relations) are: 1.161 for $90^{\circ}$ layer and 0.464 for $0^{\circ}$ layer. As expected, present RMZC model considerably improves predictions of the first-order shear-deformation formulation. Other results related to plate examples may be found in [8]. In general, RMZC results from [8] slightly differ from those presented here even for the elements of the same order. This may be due to different meshes used and due to different approaches adopted to avoid locking.

\subsection{Example 2: cylindrical bending of a shell panel}

The exact solution for the problem of simply supported cylindrical panel of infinite length, which is loaded by sinusoidally distributed pressure, was given by Ren [18]. Geometry of the panel is described by $R /$ $b=3 / \pi$, where $R$ is radius of the panel, and $b$ is its arc-length in the circumferential direction. Material properties are the same as those for example 1. The panel is loaded by a transverse pressure, sinusoidally distributed over the top surface $\bar{q}=q \sin (\pi a / b)$, where

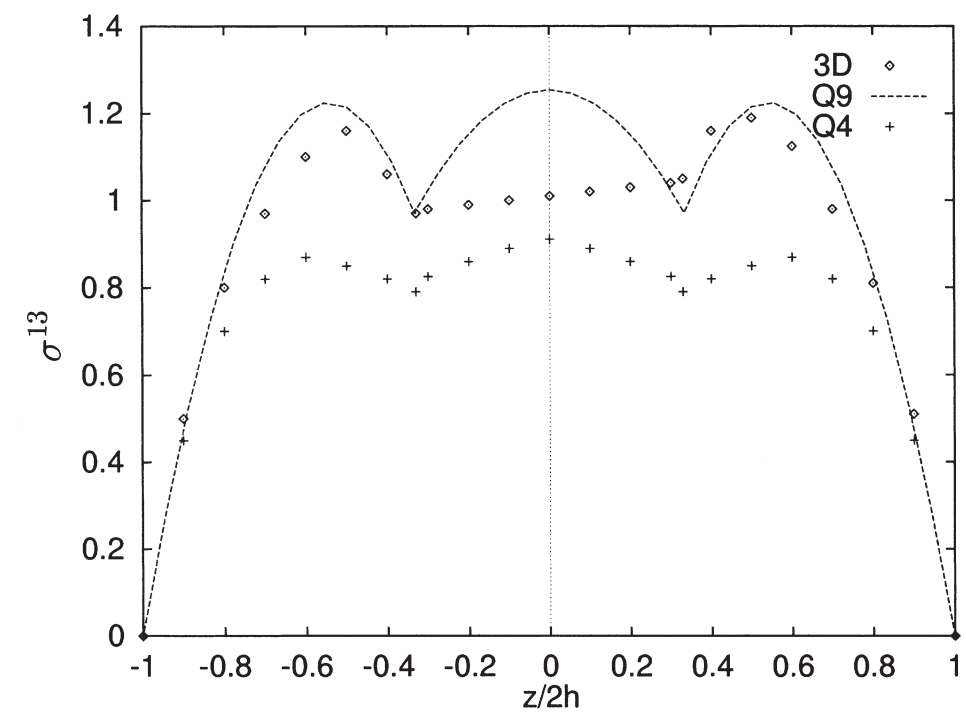

Fig. 4. Square plate. Transverse shear stress through the thickness $(z \equiv \xi)$ at closest integration point to $(0, a / 2) ; a / h=4$. 


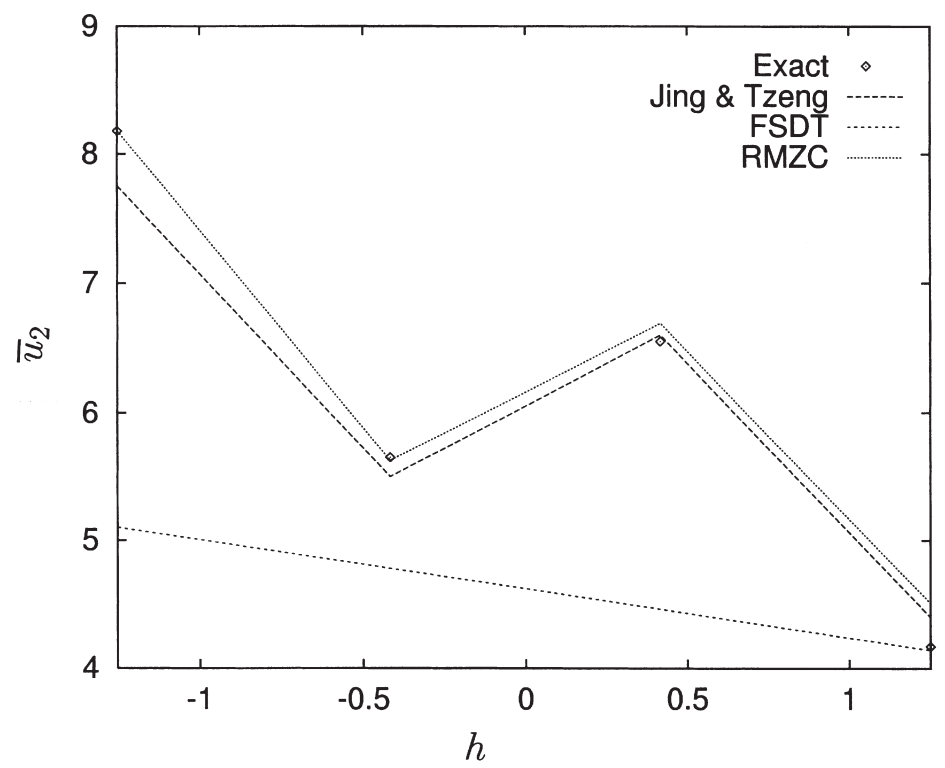

Fig. 5. Shell panel. Displacement $\bar{u}_{2}$ through the thickness $(h=2.5)$ at $a=0$ for $R / h=4$.

$a \in[0, b]$. In Fig. 5 , the normalized in-plane displacement $\bar{u}_{2}=u_{2} E_{T} h^{2} /\left(q a^{3}\right)$ at $a=0$ is plotted in the thickness direction. It can be seen that prediction of distribution of the in-plane displacement is good (results are very close to the exact analytical solution). In Table 2, transverse displacement $\bar{u}_{3}=u_{3} 10 E_{T} h^{3} /$ $\left(q R^{4}\right)$ and circumferential stress $\bar{\sigma}^{22}=\sigma^{22} h^{2} /\left(q R^{2}\right)$ are compared with the exact solution at $a=b / 2$. The same notation is used as in Section 3 and example 1, except that $\mathrm{RM}$ is first-order shear-deformation formulation with shear correction factor $\kappa=1$.

Table 2

Shell panel: present versus exact solutions; 40 elements are used for one half of a panel in circumferential direction

\begin{tabular}{|c|c|c|c|c|c|}
\hline$R / h$ & 2 & 4 & 10 & 50 & 100 \\
\hline & \multicolumn{5}{|l|}{$\bar{\mu}_{3}$} \\
\hline Exact & 1.436 & 0.457 & 0.144 & 0.0808 & 0.0787 \\
\hline RMZC & 1.5600 & 0.4656 & 0.1430 & 0.0809 & 0.0787 \\
\hline RMZ & 1.4763 & 0.4492 & 0.1400 & 0.0808 & 0.0787 \\
\hline RMC & 1.3175 & 0.3625 & 0.1226 & 0.0800 & 0.784 \\
\hline RM & 1.1988 & 0.3359 & 0.1187 & 0.0798 & 0.0784 \\
\hline \multirow[t]{2}{*}{ RMs } & 1.4175 & 0.3852 & 0.1260 & 0.0802 & 0.0785 \\
\hline & \multicolumn{5}{|c|}{$\bar{\sigma}^{22}$ at $-h / 2$} \\
\hline Exact & -3.462 & -1.772 & -0.995 & -0.798 & -0.786 \\
\hline RMZC & -2.293 & -1.440 & -0.941 & -0.795 & -0.785 \\
\hline RMZ & - & -1.442 & -0.939 & - & -0.785 \\
\hline
\end{tabular}

\section{Non-linear examples}

\subsection{Example 3: cylindrical panel under a point load}

Geometry of the panel is defined as: radius is $R=$ 12.73239, length is $L=10$, angle in circumferential direction is $\varphi=45^{\circ}$, thickness is $h=1$. Orientation of layers is $\left[45^{\circ} /-45^{\circ} / 45^{\circ}\right]$ and material characteristics are $E_{L}=40, E_{T}=1, G_{L T}=0.5, G_{T T}=0.2, v_{L T}=0.25$. The two straight edges are simply supported (i.e. $u_{3}=0$ ), while the other two edges are hinged $\left(u_{3}=u_{1}=0\right)$, where $u_{3}$ is displacement in the thickness direction and $u_{1}$ is the displacement in the circumferential direction. Point load at the middle of the panel is applied. Mesh is $10 \times 10$ elements for the full panel. Force versus displacement under the force diagram is plotted in Fig. 6 for RMC, FSDT (first-order shear-deformation) i.e. RM, and CLT (classical laminate model) element. The latter is obtained by using shear correction factor as a penalty number. Shear correction factor of FSDT element is $\kappa=1$. It can be seen that RMC and FSDT results differ only slightly. The advantage of RMC over FSDT formulation is that in the former the 'shear correction factor' is taken into account automatically by the assumed distribution of transverse shear stresses over the thickness.

\subsection{Example 4: snap-through of a cylindrical panel}

Geometry data, material characteristics and finite element mesh of this example are the same as those of example 4 , while the orientation of layers is $\left[0^{\circ} / 0^{\circ} / 0^{\circ}\right]$. 


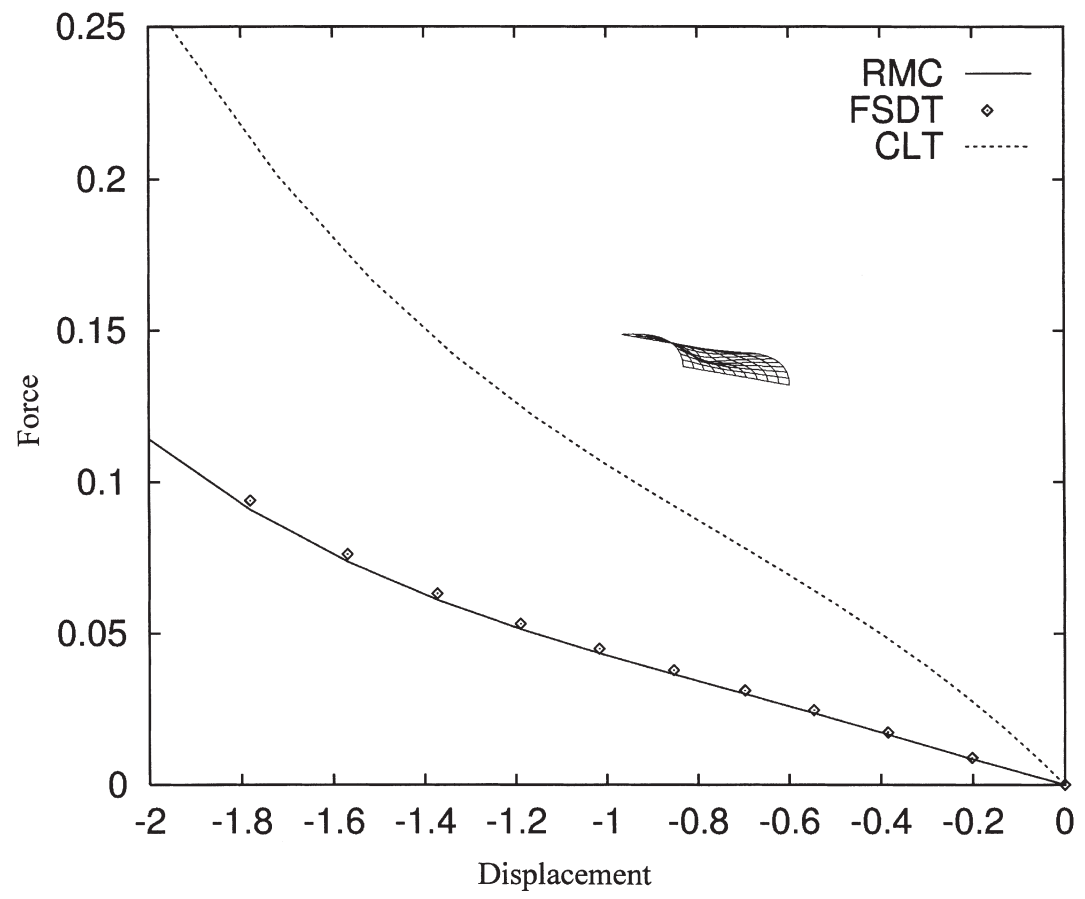

Fig. 6. Cylindrical panel; $\left[45^{\circ} /-45^{\circ} / 45^{\circ}\right]$. Force versus displacement under the force.

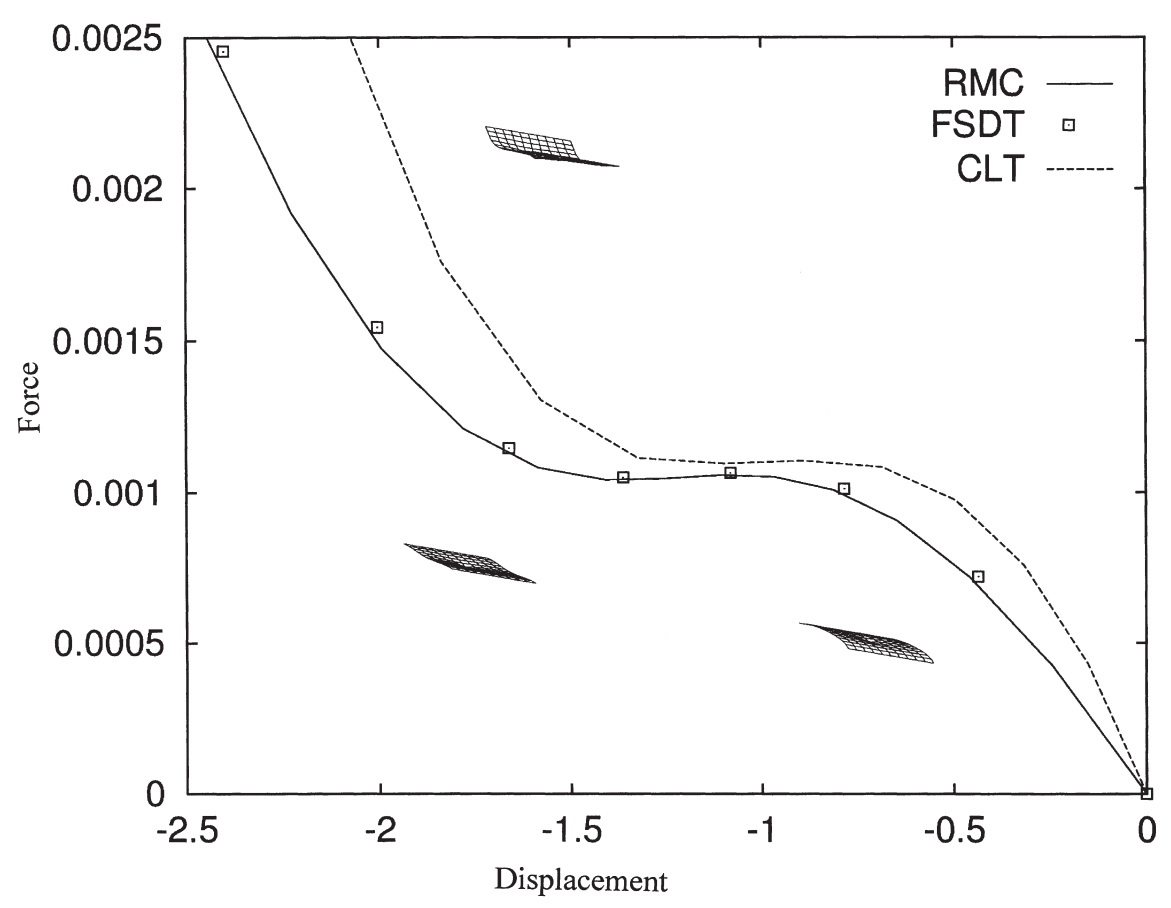

Fig. 7. Cylindrical panel; $\left[0^{\circ} / 0^{\circ} / 0^{\circ}\right]$. Force versus displacement under the force. 


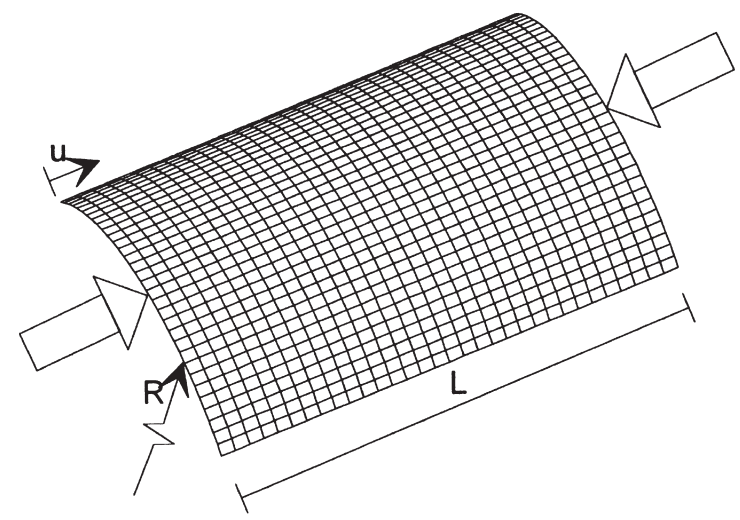

Fig. 8. Cylindrical panel: $\left[45^{\circ} /-45_{2}^{0} / 45^{\circ} / 0_{4}^{0}\right]_{s}$. Geometry, loading and finite element mesh.

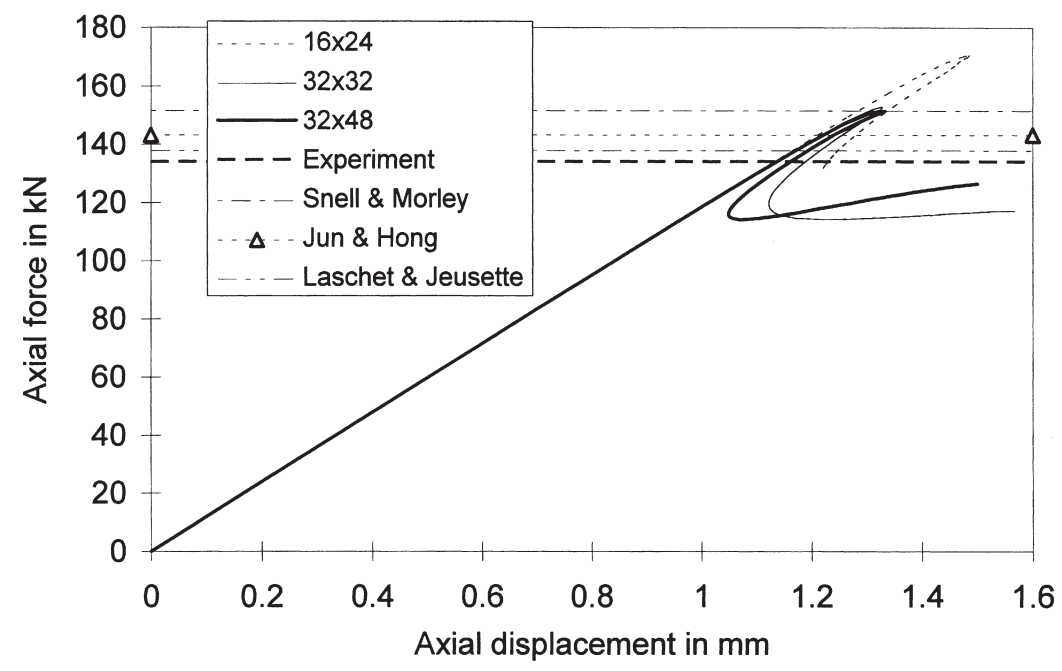

Fig. 9. Cylindrical panel; $\left[45^{\circ} /-45^{\circ}{ }_{2} / 0^{\circ}{ }_{4}\right]_{S}$. Axial force versus axial displacement.

Two straight edges are hinged $\left(u_{3}=u_{1}=0\right)$, while the displacements of curved edges are not restrained. The results of three models are shown in Fig. 7, where the diagrams of force versus displacement under the force are plotted. Again, it can be seen that the RMZC model predicts very similar global response as FSDT model.

\subsection{Examples: buckling of a cylindrical panel}

Snell and Morley [20] performed experimental and numerical tests on cylindrical panels of the following geometric data (Fig. 8): length is $L=540 \mathrm{~mm}$, thickness is $t=2.0 \mathrm{~mm}$, arc-length of curved edges is $s=$ $421.2 \mathrm{~mm}$. The panel is clamped at curved edges and simply supported at straight edges (in the direction of global coordinates). Loading was imposed by axial dis- placements at one of the curved edges. The panel is composed of 16 layers of carbon-epoxy composite material with the following set-up $\left[45^{\circ} /-45^{\circ} /-45^{\circ} /\right.$ $\left.45^{\circ} / 0_{4}\right]_{S}$. Material data is: $E_{L}=130 \mathrm{kN} / \mathrm{m}^{2}, E_{T}=10$ $\mathrm{kN} / \mathrm{m}^{2}, G_{L T}=6 \mathrm{kN} / \mathrm{m}^{2}, v_{L T}=0.3, G_{L 3}=G_{T 3}=G_{L T}$. To follow path of equilibrium configurations, modified arc-length method is used. Results, i.e. axial force versus axial displacement, are presented in Fig. 9. It can be observed, that the results for finer meshes $(32 \times 32$ elements and $32 \times 48$ elements $)$ are in agreement with the numerical prediction of Snell and Morley [20]. Better numerical results were obtained by Jun and Hon [12] and Laschet and Jeusette [13]. The difference may be due to some differences in the description of geometry and boundary conditions, which are not clearly defined in the above mentioned papers. 


\section{References}

[1] Bathe K-J, Dvorkin E. A four node plate bending element based on Mindlin/Reissner plate theory and mixed interpolation. International Journal for Numerical Methods in Engineering 1985;21:367-83.

[2] Betsch P, Menzel A, Stein E. On the parameterization of finite rotations in computational mechanics. A classification of concepts with application to smooth shells. Computer Methods in Applied Mechanics and Engineering 1998;155:273-305.

[3] Bhaskar K, Varadan TK. Reissner's new mixed variational principle applied to laminated cylindrical shells. Journal of Pressure Vessel Technology 1992;114:115-9.

[4] Brank B, Perić D, Damjanić FB. On implementation of a non-linear four-node shell finite element for thin multilayered elastic shells. Computational Mechanics 1995;16:341-59.

[5] Brank B, Perić D, Damjanić FB. On large deformations of thin elasto-plastic shells: implementation of a finite rotation model for quadrilateral shell element. International Journal for Numerical Methods in Engineering 1997;40:689-726.

[6] Brank B, Briseghella L, Tonello N, Damjanić FB. On non-linear dynamics of shells: implementation of energymomentum conserving algorithm for a finite rotation shell model. International Journal for Numerical Methods in Engineering 1998;42:409-42.

[7] Brank B, Carrera E. Multilayered shell finite element with interlaminar continuous shear stresses: a refinement of the Reissner-Mindlin formulation, 1998 (submitted for publication).

[8] Carrera E. C ${ }^{\circ}$ Reissner-Mindlin multilayered plate elements including zig-zag and interlaminar stresses continuity. International Journal for Numerical Methods in Engineering 1996;39:1797-820.

[9] Carrera E, Kröplin BH. Zig-zag and interlaminar equilibria effects in large defection and postbuckling analysis of multilayered plates. Mechanics of Composite Material and Structures 1997;4:89-94.

[10] Carrera E, Parisch H. An evaluation of geometrical nonlinear effects of thin and moderately thick multilayered composite shells. Composite Structures 1998;40:11-24.

[11] Jing H, Tzeng K. Refined shear deformation theory of laminated shells. American Institute of Aeronautics and Astronautics Journal 1993;31:765-73.

[12] Jun SM, Hong CS. Buckling behavior of composite laminated cylindrical panels and under axial compression. Computers and Structures 1998;29:479-90.

[13] Laschet G, Jeusette J-P. Postbuckling finite element analysis of composite panels. Composite Structures 1990;14:35-48.

[14] Murakami H. Laminated composite plate theory with improved in-plane response. Journal of Applied Mechanics 1986;53:661-6.

[15] Noor AK, Burton WS, Bert CW. Computational models for sandwich panels and shells. Applied Mechanics Reviews 1996;9:155-99.

[16] Pagano NJ. Exact solutions for laminates in cylindrical bending. Journal of Composite Material 1969;3:398-411.

[17] Pagano NJ, Hatfield SJ. Elastic behavior of multilayered bidirectional composites. American Institute of Aeronautics and Astronautics Journal 1972;10:931-3.

[18] Ren JG. Exact solutions for laminated cylindrical shells in cylindrical bending. Composite Sciences and Engineering 1987;29:169-87.

[19] Sansour C. A theory and finite element formulation of shells at finite deformations involving thickness change: circumventing the use of a rotation tensor. Archive of Applied Mechanics 1995;65:194-216.

[20] Snell M, Morley N. The compression buckling behavior of highly curved panels of carbon fiber reinforced plastics. In: Harrison W, Strife J, Dhinga A, editors. Proceedings of IICCM V. Warrendale, PA: Metallurgical Society, 1985. pp. 1327-53. 